Research paper

\title{
Dephosphorylated rather than hyperphosphorylated Tau triggers a pro- inflammatory profile in microglia through the p38 MAPK pathway
}

\author{
Juan Ramón Perea ${ }^{\mathrm{a}, \mathrm{b}}$, Jesús Ávila ${ }^{\mathrm{a}, \mathrm{b}}$, Marta Bolós ${ }^{\mathrm{a}, \mathrm{b}, *}$ \\ a Department of Molecular Neuropathology, Centro de Biología Molecular "Severo Ochoa", CBMSO, CSIC, Madrid, Spain \\ ${ }^{\mathrm{b}}$ Network Center for Biomedical Research on Neurodegenerative Diseases (CIBERNED), Madrid, Spain
}

\section{A R T I C L E I N F O}

\section{Keywords:}

Alzheimer's disease

Tau

Microglia

Inflammation

Mitogen-activated protein kinase (MAPK)

\begin{abstract}
A B S T R A C T
Tauopathies are a broad set of neurodegenerative dementias characterized by the aggregation of Tau protein. Activated microglia and elevated levels of pro-inflammatory molecules are also pathological hallmarks of tauopathies. In these diseases, intracellular Tau is secreted to the extracellular space, where it interacts with other cells, such as neurons and glia, promoting inflammation. However, the mechanism through which extracellular Tau triggers pro-inflammatory responses in microglia remains unknown. Primary microglia cultures were treated with extracellular Tau in its hyperphosphorylated, dephosphorylated or non-phosphorylated form. Protein cytokine arrays, real-time PCR, inhibition of the p38 MAPK pathway, phosphatase assays, and quantification of proteins through immunoblotting were used to analyze the effect of extracellular Tau on the proinflammatory response of microglia. The main finding of this work is that extracellular non-phosphorylated and dephosphorylated forms of Tau, rather than hyperphosphorylated Tau, activate the p38 MAPK pathway in microglia, thus triggering a pro-inflammatory response in these cells.
\end{abstract}

\section{Introduction}

The abnormal accumulation of intracellular hyperphosphorylated Tau protein is the common pathological hallmark of a set of neurodegenerative diseases collectively referred to as tauopathies (Medina et al., 2016). Alzheimer's disease (AD) is the most common form of tauopathy. $\mathrm{AD}$ is a fatal neurodegenerative disorder characterized by progressive cognitive and functional impairment, and memory loss (Whitehouse et al., 1982). In the AD brain, Tau is hyperphosphorylated, meaning that it is phosphorylated to a higher degree at physiological sites, as well as at pathological sites (Bussiere et al., 1999; Hasegawa et al., 1996; Hoffmann et al., 1997; Jicha et al., 1997). Together with amyloid- $\beta$ (A $\beta$ ) plaques, Tau aggregates (neurofibrillary tangles (NFTs)) are the defining histopathological hallmarks of AD (Selkoe et al., 1982; Tomlinson et al., 1970).

Recent research has shown that the most toxic form of Tau is not NFTs themselves, but rather the smaller aggregates, called Tau oligomers, which are likely to initiate neurodegeneration in tauopathies (Andorfer et al., 2005; Cowan and Mudher, 2013; Fox et al., 2011; Ishihara et al., 1999; Ishihara et al., 2001; Le Corre et al., 2006; SpiresJones et al., 2009; Wittmann et al., 2001). Intracellular Tau can be released into the extracellular space (Perez et al., 2016; Yamada et al., 2014) and can affect neighboring cells, such as neurons (Gomez-Ramos et al., 2006) and glia (Bolos et al., 2015; Leyns and Holtzman, 2017). In this regard, we have recently shown that extracellular soluble Tau, mainly composed of monomers and dimers, has devastating effects on the structural plasticity of hippocampal granule neurons (Bolos et al., 2017b). This finding thus supports the notion that impaired clearance of extracellular Tau contributes to the progression of $\mathrm{AD}$ (Alonso et al., 2016; Hyman, 2014; Medina and Avila, 2014a, 2014b; Mirbaha et al., 2015).

Microglia are the resident immune cells in the brain, and they participate in neuroprotection and the maintenance of homeostasis (Bolos et al., 2018; Ransohoff and El Khoury, 2016). Under pathological conditions, microglia are activated and become ramified. These cells have the capacity to proliferate, migrate, and efficiently phagocytose pathogens and cellular debris (Aloisi, 2001; Diaz-Aparicio et al., 2016; Kreutzberg, 1996; Sierra et al., 2014). Furthermore, activated microglia can release a host of pro-inflammatory cytokines, including interleukin (IL) 1-beta (IL-1 $\beta$ ), tumor necrosis factor alpha (TNF- $\alpha$ ), IL-6, IL-18, and interferon gamma (IFN- $\gamma$ ), among others (Gonzalez-Scarano and Baltuch, 1999; Hanisch, 2002; Ransohoff, 2016).

Mitogen-activated protein kinases (MAPKs) are members of specific signaling cascades that serve as convergent points for numerous and diverse extracellular signals and are thus critical integrators of signaling events (Arthur and Ley, 2013; Bradham and McClay, 2006). Recent

\footnotetext{
* Corresponding author at: Department of Molecular Neuropathology, Centro de Biología Molecular "Severo Ochoa", CBMSO, CSIC, Madrid, Spain.

E-mail address: mbolos@cbm.csic.es (M. Bolós).
} 
years have witnessed increasing interest in p38 MAPKs (p38) in the AD research field. Given their involvement in inflammation and cell death pathways, these kinases may contribute to the pathogenic events that occur in the AD brain (Correa and Eales, 2012; Sun et al., 2003; Wang et al., 2014; Zhu et al., 2002). Several studies report the relationship between $A \beta 1-42$, mainly oligomers, and p38 activation in microglia (Bachstetter et al., 2011; Lee and Kim, 2017). The increased levels of oligomeric $A \beta$ in the $A D$ brain may activate signaling cascades that increase p38 activity (Adolfsson et al., 2012). However, the effect of Tau on p38 activation and its contribution to microglia-mediated inflammation remain unknown.

Here we show that extracellular Tau modulates the pro-inflammatory profile of microglia by regulating p38 activity in these cells. Furthermore, this effect was produced only when Tau was dephosphorylated, while it was absent when this protein was hyperphosphorylated.

\section{Results}

\section{1. p38 MAPK is a target of Tau in microglia}

We previously reported that extracellular Tau is internalized by microglia (Bolos et al., 2015; Bolos et al., 2017a). However, the effect of Tau on these cells remains unclear. In $\mathrm{AD}$, which is also considered as an inflammatory disease (Bolos et al., 2017c; McGeer et al., 1996), A $\beta$ activates p38 (Culbert et al., 2006; McDonald et al., 1998). Therefore, we studied whether Tau modulates p38 expression in these cells. To this end, primary microglia cultures were treated with Tau derived from $E$. coli (non-phosphorylated (npTau)) at $1 \mu \mathrm{M}$ or vehicle (phosphate saline buffer (PBS)) for $0,30,120$, or $240 \mathrm{~min}$. The phosphorylation of p38 in Thr180/Tyr182 residues, which implies kinase activation, was analyzed as (p-p38/GAPDH)/ (p38/GAPDH). An increase in phosphorylated p38 (p-p38) was observed after 30 min of treatment with non-phosphorylated Tau (npTau) (Fig. 1A-B). In contrast, this effect was not replicated when cells were treated with PBS under the same conditions (Fig. 1A-B). Although there was an increase in p-p38 after treatment with npTau at the other times tested, the differences, as shown by 2way ANOVA, were not statistically significant when compared with the control cells treated with PBS. Therefore, 30 min of treatment was used in the rest of the experiments.

To study whether this effect was replicated in vivo, 2-month-old C57Bl/6 mice were stereotaxically injected with npTau or PBS (Fig. 1C). After 2 weeks, the amount of p-p38, analyzed following the same protocol as for in vitro experiments, was increased in the brain of these mice compared to control ones injected with PBS (Fig. 1D-E). However, to confirm that the activation of p38 was an effect mediated by Tau specifically in microglial cells, which was the main objective of this work, we performed the rest of the experiments using an in vitro model of microglial primary cultures because the tissue derived from brain contains other types of cells.

\subsection{Hyperphosphorylated Tau has no effect on p38 in microglia}

In tauopathies such as $\mathrm{AD}$, Tau is hyperphosphorylated (GrundkeIqbal et al., 1986; Medina and Avila, 2014a). Due to the importance of this posttranslational modification of this protein in these diseases, the activation of p38 (p-p38) in microglia was tested in the presence of hyperphosphorylated Tau (hypTau), which was isolated from sf9 cells (Gomez-Ramos et al., 2004). We observed that npTau activated p38 in microglia, as described above (Fig. 2A-B). However, p38 was not activated in the presence of hypTau. Therefore, we next examined the difference, in terms of phosphorylation, between the two forms of Tau using the PHF-1 antibody (which recognizes S396/S404 phosporylated residues of Tau) (Fig. 2C). The western blot showed that there was no

A

B
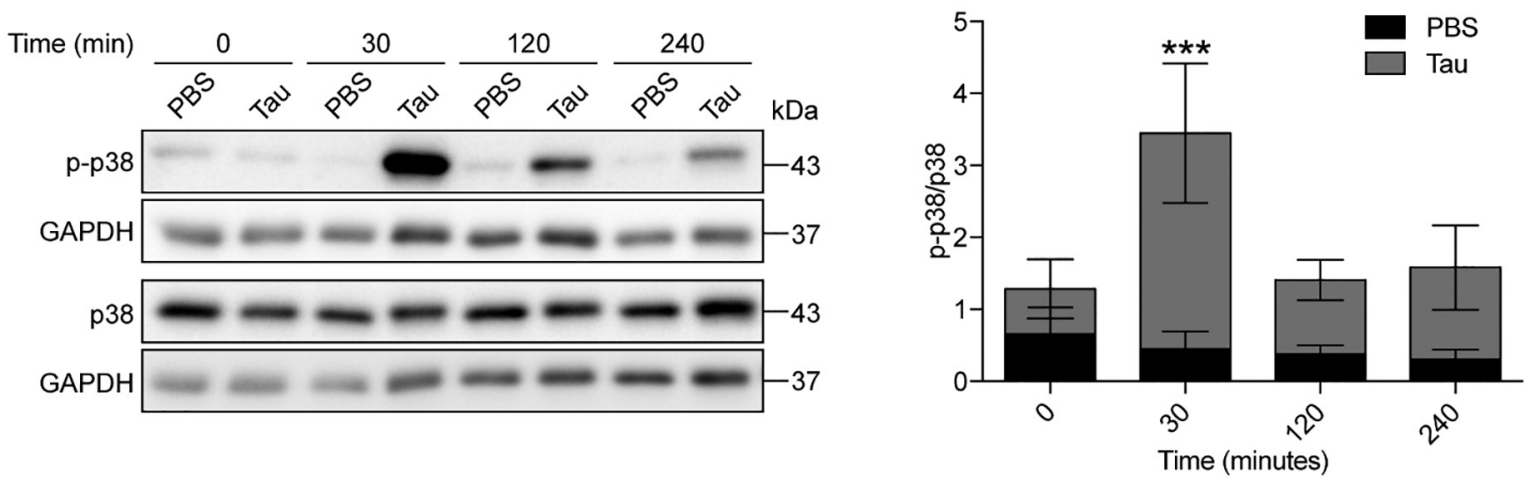

C

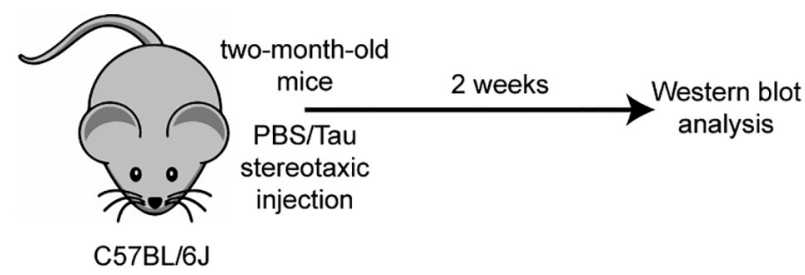

D

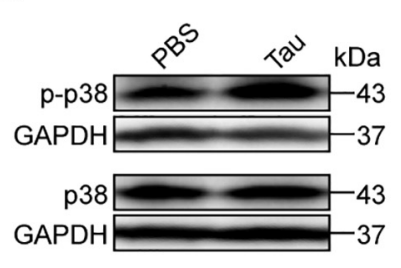

E

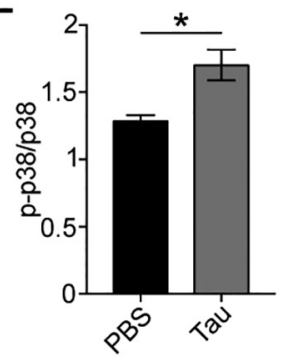

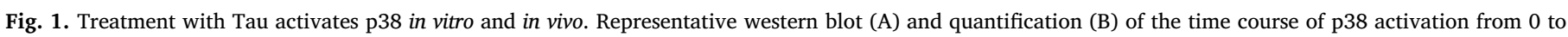

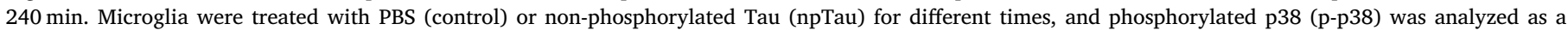

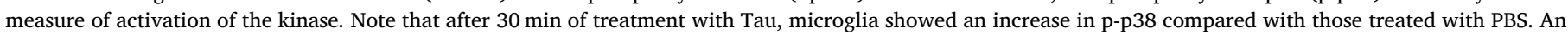

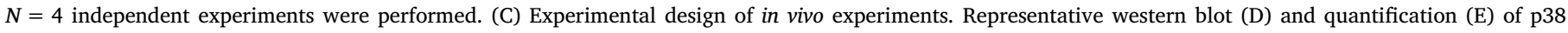
activation in the tissue of injected mice. Bars show means \pm S.E. $* p \leq 0.05$, $* * * p \leq 0.001$. 
A

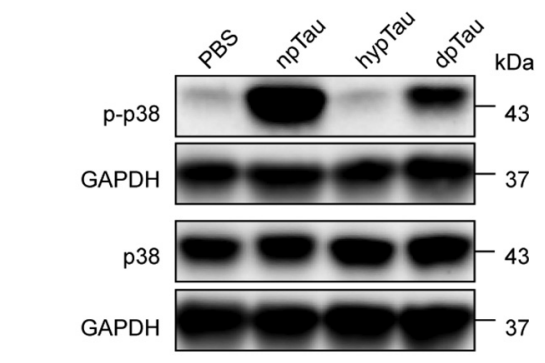

B

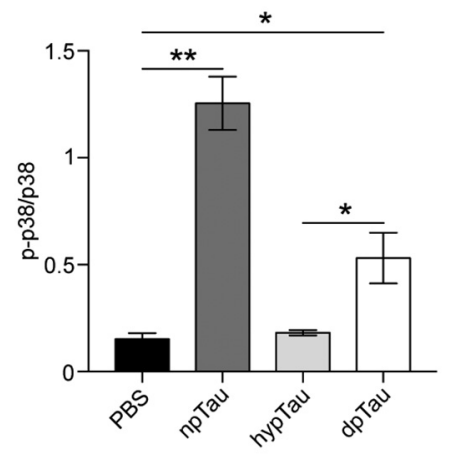

C

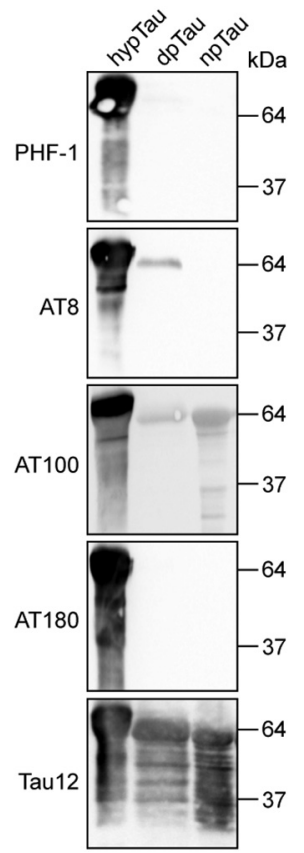

Fig. 2. Hyperphosphorylated Tau does not activate p38 in microglia. (A) Western blot and (B) quantification of phosphorylated p38 (p-p38) and p38 from microglia samples after treatment with PBS, non-phosphorylated Tau (npTau), hyperphosphorylated Tau (hypTau) or dephosphorylated Tau (dpTau) (meaning hypTau after treatment with $\lambda$-phosphatase). An $N=3$ independent experiments was performed. (C) Western blot of phosphorylated Tau in residues measured by PHF-1, AT8, AT100 and AT180 antibodies of hypTau, dpTau and npTau samples. Total Tau was measured by Tau12 antibody. Note that only hypTau was phosphorylated in several residues. Bars show means \pm S.E. $* p \leq 0.05, * * p \leq 0.01$.

PHF-1 signal in npTau (Fig. 2C, third lane). As expected, hypTau was stained with the antibody (Fig. 2C, first lane). The total amount of Tau protein was measured using Tau12 antibody, checking that there was

no difference between samples (Fig. 2C first and third lanes). To confirm that npTau was not phosphorylated in residues other than those stained by the PHF-1 antibody, we tested the AT8, AT100 and AT180 antibodies (which indicate phosphorylation at S202/T205, T231 and T212/S214, respectively) (Fig. 2C). HypTau showed a positive signal for the three antibodies; however, as expected, npTau did not (Fig. 2C, first and third lanes). Therefore, to examine whether p38 was activated only in the presence of Tau without phosphorylated residues, hypTau was dephosphorylated by means of lambda phosphatase ( $\lambda$-phosphatase) treatment, as indicated in material and methods section, obtaining dephosphorylated Tau (dpTau). No PHF-1 signal was detected for dpTau (Fig. 2C, second lane). Furthermore, this form of Tau was not phosphorylated in other residues, as shown by the AT8, AT100 and AT180 antibodies (Fig. 2C, second lanes). These results confirmed that hypTau was totally dephosphorylated by $\lambda$-phosphatase treatment. Finally, microglia were incubated with dpTau (hyperphosphorylated Tau $+\lambda$-phosphatase) and p-p38 was analyzed. We observed that dpTau activated p38 (Fig. 2A-B). These results confirmed that p38 was activated only in the presence Tau free of phosphorylated residues. Therefore, we performed the rest of the work with npTau, referred to here after simply as Tau, because it was not phosphorylated basally and was more easily obtained from a technical point of view than dpTau.

\subsection{Tau modulates the p38 MAPK pathway in microglia}

Next, to confirm the direct effect of the presence of Tau on the activation of the p38 pathway in microglia, an inhibitor of p38, named SB203580 (SB), was used (Piao et al., 2003). Microglia cultures were pre-treated with $20 \mu \mathrm{M}$ of SB for $30 \mathrm{~min}$ before $1 \mu \mathrm{M}$ of Tau or PBS (control) was added to the cells for another $30 \mathrm{~min}$. After this time, the immunoblotting analysis showed that p38 was phosphorylated by the treatment of Tau, independently of the presence or absence of SB (Fig. 3A-B). This finding can be explained by the fact that SB inhibits the catalytic activity of $\mathrm{p} 38$ by binding to the ATP binding pocket, but it does not block the phosphorylation of p38 by upstream kinases (Young et al., 1997). Given this explanation, we studied the activation of Mitogen-activated protein kinase-activated protein kinase 2 (MK2)-a kinase protein downstream of p38-in Thr334 residue, as an indirect measure of p38 activity in microglia (Ghasemlou et al., 2010). In
A

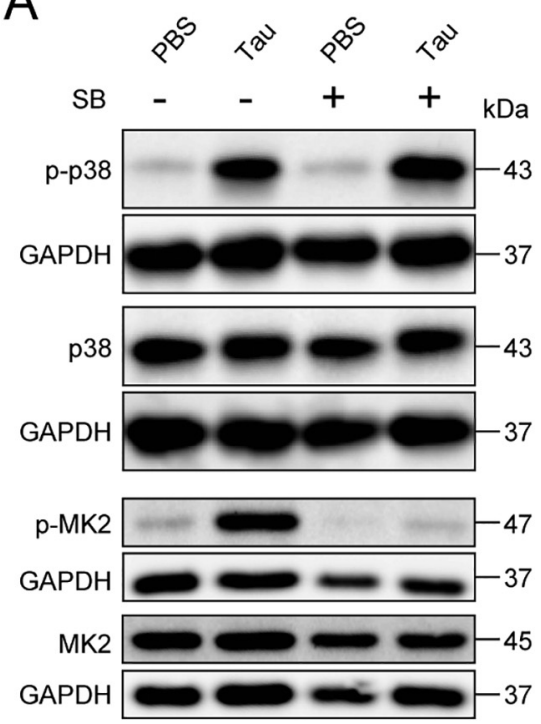

B
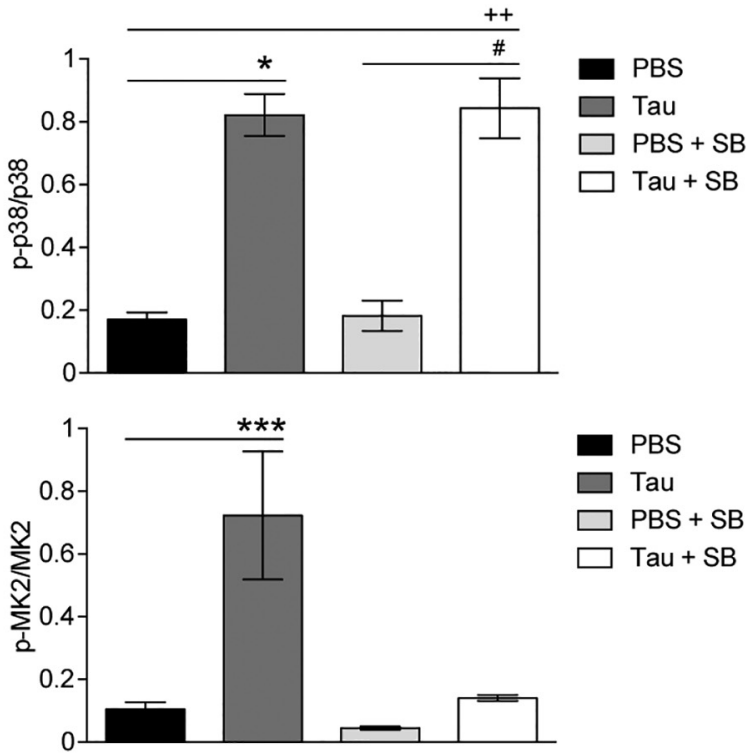

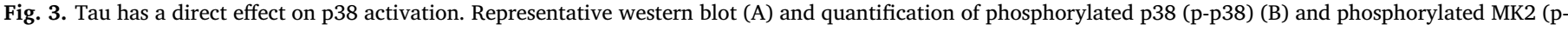

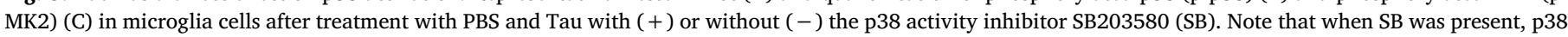

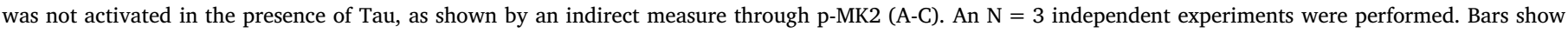
means \pm S.E. $* p \leq 0.05, \# p \leq 0.05,++p \leq 0.01$, and $* * * p \leq 0.001$. 
microglia cultures treated with Tau, the activation of $\mathrm{p} 38$ induced MK2 phosphorylation, showed as phosphorylated MK2 (p-MK2) (Fig. 3A-C). However, this effect was not observed after PBS treatment (Fig. 3A-C). Furthermore, when microglia were pre-treated with SB and Tau was then added to the cells, MK2 was not phosphorylated since p38 activity was inhibited (Fig. 3A-C). These results confirmed that the presence of Tau activated p38 in microglia. In addition, in the presence of SB, although p38 was phosphorylated, its activity was impaired when cells were treated with Tau.

\subsection{Tau produces a pro-inflammatory profile in microglia}

We next studied the effects of Tau on the early response of microglial cells. To this end, microglia primary cultures were treated with $1 \mu \mathrm{M}$ of Tau or PBS. Cell media were collected, and a cytokine protein array on media samples was performed. Fig. 4A shows the cytokines whose levels displayed changes of $>25 \%$ with respect to their levels in the PBS control treatment (dotted line). The expression of the remaining cytokines is shown in Supporting Fig. S1. The levels of some of the most widely characterized pro-inflammatory cytokines, such as IL6 , -9 , and -17 , among others, and macrophage inflammatory protein (MIP) $1 \alpha$ were markedly increased in the media from Tau-treated microglia compared to control media from microglia treated with PBS (Fig. 4A). These results suggested that the treatment with Tau induced a pro-inflammatory response in microglia and, consequently, the secretion of pro-inflammatory cytokines by these cells.

\section{5. npTau triggers a pro-inflammatory profile in microglia through the $p 38$ MAPK pathway}

To confirm the pro-inflammatory response of microglia after Tau treatment, two of the cytokines that were increased in the culture medium, as shown by protein array, were selected, such as Il6 and Mip $1 \alpha$, and their synthesis by microglia was confirmed using the qPCR approach. The results were consistent with those obtained in the cytokine protein array. Microglia treated with npTau showed an increase in Il6 and Mip1 $\alpha$ expression (Fig. 4B). In addition, as a positive control, other well-known genes related to the inflammatory response of microglia, such as Il1b and Tnfa, were also analyzed. Although the protein levels of these two cytokines were not increased, as determined by the cytokine array (Fig. 4A), gene expression levels were increased after treatment with npTau, as shown by qPCR. However, this experiment performed with hypTau did not show the same increased level in cytokine expression as that observed with npTau (Fig. 4B). The levels of gene expression were represented relative to the expression levels in microglia treated with PBS.

Next, we studied whether there was a link between Tau treatment and cytokine expression through the activation of p38 in microglia. To address this question, microglia cultures were pre-treated with SB for $30 \mathrm{~min}$ before the addition of $1 \mu \mathrm{M}$ of npTau or hypTau. Following the same protocol, the expression of Il6, Mip $1 \alpha, I l 1 b$ and Tnf $\alpha$ was analyzed by qPCR. The expression of all these kinases was significantly reduced compared to control cells (npTau treatment) in the presence of SB (Fig. 4C). This result confirmed that Tau triggered the expression of proinflammatory cytokines through the activation of p38 in microglia.

\section{Discussion}

Here we reveal the following: 1) npTau and $\lambda$-phosphatase-treated Tau, which encompasses dpTau, induce the activation of the p38 pathway in microglia; 2) microglial p38 is not activated when Tau is hyperphosphorylated; 3) p38 activation in microglia by Tau treatment leads to an overproduction of pro-inflammatory cytokines; and, 4) inhibition of the p38 pathway suppresses the inflammatory response mediated by microglia in the presence of Tau.

Tau is a microtubule-binding protein that participates in
A

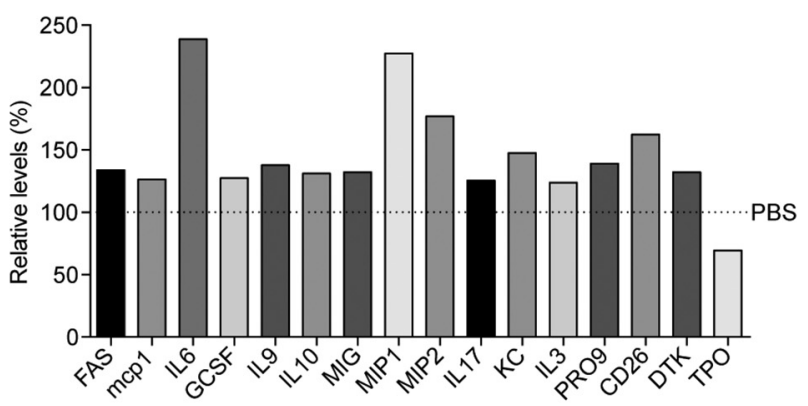

B

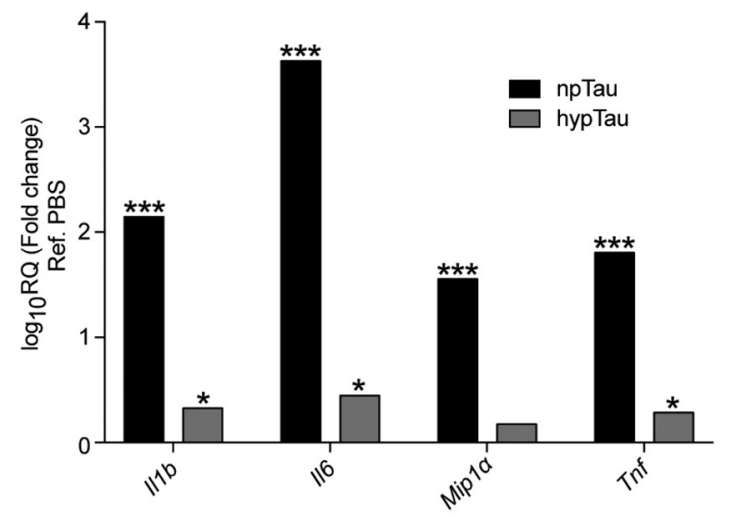

C

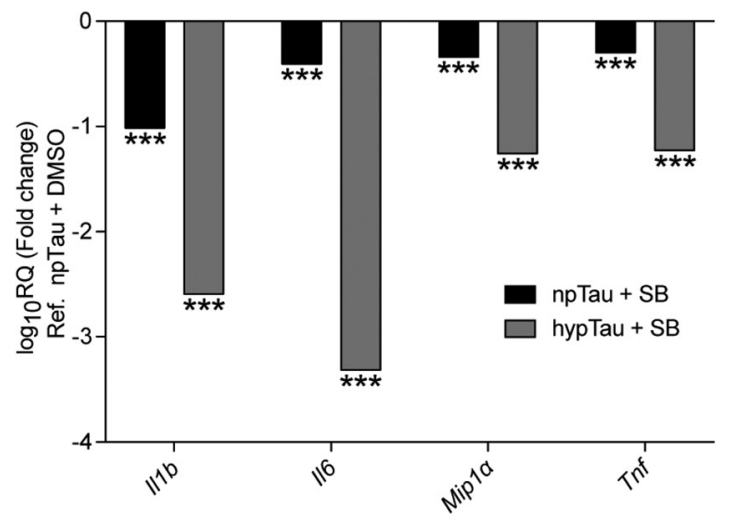

Fig. 4. Tau treatment induces a pro-inflammatory profile in microglia. (A) Cytokine protein array in the culture medium of microglia after treatment with Tau or PBS. Only those cytokines whose levels showed changes $>25 \%$ with respect to those of PBS (dotted line) treatment are shown (the rest of the cytokines analyzed are indicated in Supporting Fig. S1). Relative levels of expression are shown. (B-C) Real-time PCR of Il1b, Il6, Mip1 $\alpha$ and Tnf genes in microglia cultures treated with npTau and hypTau in the presence (B) or absence (C) of SB. $\mathrm{N}=3$ independent experiments were performed.

microtubule assembly and dynamics processes that may regulate neuron morphology (Caceres and Kosik, 1990; Drubin and Kirschner, 1986), among others. The abnormal accumulation of Tau protein in intracellular aggregates is observed across a broad spectrum of neurodegenerative disorders collectively referred to as tauopathies (Medina et al., 2016). Posttranslational modifications, such as phosphorylation, in specific residues of Tau are crucial for its physiological and pathological activities (Avila et al., 2016). Although considered a histopathological hallmark in tauopathies, intraneuronal NFTs, which are formed by hypTau (Selkoe et al., 1982), do not appear to be the main toxic entities leading to disease (Gerson et al., 2014). Indeed, recent studies suggest that more soluble forms of Tau rather than fibrillar tangles are involved in toxicity and the spread of the disease (Fa et al., 2016; Gerson et al., 2014; Gomez-Ramos et al., 2006; Haass and Selkoe, 2007). 
In these diseases, Tau inclusions have also been reported in microglia (Leyns and Holtzman, 2017; Odawara et al., 1995), despite their lack of Tau expression, thus providing further evidence that pathological Tau is also transferred between brain cell populations (Holmes and Diamond, 2014; Hyman, 2014; Maphis et al., 2015; Medina and Avila, 2014a, 2014b).

Tau secreted to the extracellular space, via various mechanisms, is dephosphorylated by tissue-nonspecific alkaline phosphatase (TNAP) (Diaz-Hernandez et al., 2015). This dpTau behaves as a muscarinic receptor agonist, its activation causing a robust and sustained increase in intracellular calcium that finally leads to neuronal death (DiazHernandez et al., 2010; Gomez-Ramos et al., 2006). Additionally, we have recently described that npTau has devastating effects on the structural plasticity of hippocampal newborn neurons (Bolos et al., 2017b). Furthermore, a more recent study showed that Tau clusters in hippocampal and cortical regions of aged wild-type mice. This observation thus supports the accumulation of dpTau as a plausible earlystage indicator of neuronal damage and/or vulnerability that precedes overt NFT formation (Tseng et al., 2017). Here we have demonstrated that npTau and dpTau activates the $\mathrm{p} 38$ pathway in microglia and that this effect is not exerted by hypTau. Our novel results, together with these studies, support the notion that extracellular dpTau is one of the triggers of inflammation in early stages of AD. Further studies are needed to elucidate the mechanism through which Tau triggers the cascade that results in p38 activation in microglia.

Brain inflammation is one of the most important risk factors for the development of sporadic AD (Bolos et al., 2017c). The inflammatory response is driven mainly by activated microglia (Ransohoff, 2016) and is accompanied by increased levels of specific chemokines and cytokines. A $\beta$-induced expression of pro-inflammatory cytokines in microglial cells causes neuroinflammation, and this action is mediated by $\mathrm{p} 38$ (Bachstetter et al., 2011; Munoz et al., 2007; Roy et al., 2015; Watterson et al., 2013). p38-mediated cytokine overproduction in microglia has been linked to inflammation-induced neurotoxicity in a variety of mouse models (Bachstetter et al., 2014; Bachstetter et al., 2011; Barone et al., 2001a; Correa and Eales, 2012; Dewil et al., 2007; Xing et al., 2011). In this regard, we have seen that microglial p38 activation after treatment with npTau led to the overexpression of several pro-inflammatory cytokines, such as IL- 6 and MIP1 $\alpha$, at the protein and gene expression level. Although hypTau increased the expression of some cytokines, the effect was almost insignificant when compared to that of npTau. Furthermore, when a well-known p38 inhibitor (SB203580) (Barone et al., 2001b; Piao et al., 2003; Underwood et al., 2000) was used, the production of these pro-inflammatory cytokines was abolished. These studies support targeting the p38 signaling pathway as a potential strategy to suppress the innate immune responses of microglia and prevent inflammation (Lee and Kim, 2017; Scheltens et al., 2018; Watterson et al., 2013).

In summary, here we show, for the first time, that extracellular npTau and dpTau lead to the activation of the p38 pathway in microglia, thus triggering a pro-inflammatory response in these cells. On the basis of this finding, we propose that the hyperphosphorylation of Tau abolishes the activation of p38 and the subsequent inflammation in microglia. Therefore, extracellular dpTau could be more toxic and pathogenic than previously believed and thus could be a crucial contributor to the spread of the disease. These findings pave the way to new therapies based on removing the extracellular source of dpTau, instead of the most commonly used antibody therapy focused on hypTau.

\section{Materials and methods}

\subsection{Animals}

C57Bl/6 mice pups (from the CBMSO animal facility) were used for the microglia primary culture experiments. In addition, 2-month-old
C57Bl/6 (Harlan Laboratories, The Netherlands) mice were used for the in vivo experiments involving stereotaxic injection of Tau or PBS. Mice were housed in a specific pathogen-free colony facility, in accordance with European Community Guidelines (directive 86/609/EEC), and handled following European and local animal care protocols. Animal experiments were approved by the CBMSO's Ethics Committee and the National Ethics Committee (AEEC-CBMSO-62/14).

\subsection{Human Tau proteins}

The recombinant human Tau isoform, containing $2 \mathrm{~N}$-terminal inserts and 4 microtubule binding repeats (Avila et al., 2016), was isolated from Escherichia coli (Medina et al., 1995) (named non-phosphorylated Tau (npTau) in this work) with some modifications. IPTGinduced $E$. coli pellets were sonicated in extraction buffer (0.1 M MES; $2 \mathrm{mM}$ EGTA; $0.5 \mathrm{mM} \mathrm{MgCl}_{2} ; 0.5 \mathrm{M} \mathrm{NaCl} ; 5 \mathrm{mM} \beta$-mercaptoethanol; $1 \mathrm{mM}$ PMSF). Sonicated sample was centrifuged ( $10 \mathrm{~min}, 13,000 \mathrm{rpm}$, $4{ }^{\circ} \mathrm{C}$ ) and the supernatant was boiled for $10 \mathrm{~min}$ to prevent possible contamination of endogenous enzymatic activities. Subsequently, the boiled sample was incubated for $5 \mathrm{~min}$ on ice and then centrifuged (30 min, $13,000 \mathrm{rpm}, 4^{\circ} \mathrm{C}$ ). Then, Tris was added to the supernatant until it reaches a $\mathrm{pH}$ of 11 . To induce Tau precipitation, sample was stirred in a magnetic mixer for $1 \mathrm{~h}$ at $4{ }^{\circ} \mathrm{C}$ and $\left(\mathrm{NH}_{4}\right)_{2} \mathrm{SO}_{4}$ was added to the sample $(50 \%$ saturation). Next, sample was centrifuged $(1 \mathrm{~h}$, $13,000 \mathrm{rpm}, 4^{\circ} \mathrm{C}$ ) and pellet was resuspended in PBS. After that, resuspended pellet was dialyzed overnight at $4{ }^{\circ} \mathrm{C}$ (Spectra/Por ${ }^{\circledR} 3$ Dialysis membrane, Sigma, USA) in PBS. Then, each $\mathrm{ml}$ of dialyzed sample was incubated for $1 \mathrm{~h}$ at $37^{\circ} \mathrm{C}$ at $300 \mathrm{rpm}$ with $1 \mu \mathrm{l}$ of RNase A (Roche, Switzerland). In order to stop RNase enzymatic activity and also remove it, sample was incubated for $1 \mathrm{~min}$ at $100^{\circ} \mathrm{C}$ and finally centrifuged ( $1 \mathrm{~min}, 13,000 \mathrm{rpm}, 4^{\circ} \mathrm{C}$ ). Supernatant was pooled as tau fractions and stored frozen until use.

Sf9 cells infected with baculovirus that contains a recombinant bacmid DNA with the human Tau cDNA were also used to isolate recombinant human Tau (named hyperphosphorylated Tau (hypTau) in this work) (Gomez-Ramos et al., 2004). Briefly, Sf9 cells were grown at $27^{\circ} \mathrm{C}$ in a spinner culture bottle in Grace's Insect Medium (Gibco, USA), supplemented with $10 \%$ fetal bovine serum (Gibco, USA), $10 \mathrm{U} / \mathrm{ml}$ of penicillin (Pancreac, Spain), $0.1 \mathrm{mg} / \mathrm{ml}$ of streptomycin (Pancreac, Spain), $200 \mu \mathrm{g} / \mathrm{ml}$ of Ciprofloxacin (Sigma, USA) and $0.05 \%(v / \mathrm{v})$ Pluronic F68 (Gibco, USA). Monolayer cultures of Sf9 cells were infected with recombinant baculovirus at a multiplicity of infection of $1-5$. Samples were taken at $72 \mathrm{~h}$ post-infection, cells were centrifuged and pellet was sonicated in extraction buffer (100 mM Tris-HCl; $0.5 \mathrm{mM}$ $\mathrm{MgCl}_{2} ; 1 \mathrm{mM}$ EGTA; $100 \mathrm{mM} \mathrm{NaCl} ; 1 \mathrm{mM}$ PMSF; $2.5 \mathrm{mM}$ Pyrophosphate; $10 \mathrm{mM}$ Glycerolphosphate; $1 \mathrm{mM}$ Sodium Orthovanadate; 100 $\mu \mathrm{M}$ Okadaic Acid; protease inhibitor mixture (Roche, Switzerland)) and centrifuged ( $30 \mathrm{~min}, 14,000 \mathrm{rpm}, 4^{\circ} \mathrm{C}$ ). Following steps are the same as those made with Tau purified from $E$. coli.

\subsection{Tau protein dephosphorylation by $\lambda$-phosphatase}

Lambda protein phosphatase is a $\mathrm{Mn}^{2+}$-dependent protein phosphatase. To obtain dpTau, this protein was used to remove phosphate groups from serine, threonine and tyrosine residues present in hypTau (Fig. 2). hypTau was dephosphorylated using a $\lambda$-phosphatase kit (Sigma, USA) following manufacturer's instructions. Briefly, hypTau was incubated with the kit's buffer plus $0.5 \mathrm{mM} \mathrm{MgCl}_{2}, 2 \mathrm{mM}$ EGTA and $0.1 \mathrm{M} \mathrm{MES}$ at $\mathrm{pH}=6.7$ for $5 \mathrm{~h}$ at $30^{\circ} \mathrm{C}$. The reaction was stopped by the addition of $50 \mathrm{mM}$ EDTA for $60 \mathrm{~min}$ at $65^{\circ} \mathrm{C}$. It was then centrifuged at $14,000 \mathrm{rpm}$ for $15 \mathrm{~min}$ at room temperature, and the supernatant was collected. Dephosphorylation of Tau was corroborated by western blot (Fig. 2B, C). Here we refer to dephosphorylated Tau as dpTau in order to differentiate from npTau, which was basally dephosphorylated. 


\subsection{Primary cultures}

Microglia were cultured from the cerebral cortices of 2-day-old C57Bl/6 mice, as described previously (Bolos et al., 2017a). Briefly, cortices were dissected, stripped of meninges, and digested with $0.1 \%$ trypsin at $37^{\circ} \mathrm{C}$ in HBSS (Gibco, USA) medium for $10 \mathrm{~min}$. Trypsinization was stopped by the addition of DMEM/Glutamax 1\%/Pyruvate $1 \%$ (Gibco, USA) culture medium supplemented with $10 \%$ fetal bovine serum (Gibco, USA), $100 \mathrm{U} / \mathrm{ml}$ of penicillin and $0.1 \mathrm{mg} / \mathrm{ml}$ of streptomycin (Gibco, USA). A single-cell suspension of the digested tissue was obtained by repeated pipetting. Cells were seeded into a 75-ml flask and cultured in medium at $37{ }^{\circ} \mathrm{C}$ in humidified $5 \% \mathrm{CO} 2-95 \%$ air. Floating microglia were recovered after 10 days by mild shaking and centrifugation. These cells were then seeded onto 24 -well plates $\left(6 \times 10^{4}\right.$ cells/well) with coverslips. Adherent cells were incubated for $48 \mathrm{~h}$ before being used for the experiments.

\section{5. p38 MAPK pathway activation assay}

Primary microglia cultures were treated with either $1 \mu \mathrm{M}$ of npTau or PBS for $0,30,120$ or 240 min or with npTau, hypTau, dpTau or PBS for $30 \mathrm{~min}$. In previous studies, we observed an effect of a Tau concentration of $1 \mu \mathrm{M}$ on microglia and also on neurons (Bolos et al., 2015; Bolos et al., 2017a; Bolos et al., 2017b; Gomez-Ramos et al., 2006). In the case of p38 MAPK inhibition experiments, microglia cultures were pre-treated with $20 \mu \mathrm{M}$ of 4-(4-fluorophenyl)-2-(4-methylsulfinylphenyl)-5-(4-pyridyl) imidazole (SB203580) (Sigma, USA) or vehicle Dimethyl Sulfoxide (DMSO) (Sigma, USA) for $30 \mathrm{~min}$ before treatment with npTau or PBS, as described above. Afterwards, cells were washed three times with PBS in order to remove excess Tau attached to the membrane. They were then lysed for the immunoblotting analysis.

\subsection{Stereotaxic surgery, sacrifice, and sample preparation}

Mice were anesthetized with Isoflurane and placed in a stereotaxic frame. Coordinates $(\mathrm{mm})$ relative to the Bregma in the anteroposterior, mediolateral, and dorsoventral planes were as follows: hippocampus $[-2.0 ; \pm 1.4 ;-2.2]$. Next, $2 \mu \mathrm{l} /$ hippocampus of npTau $(1 \mathrm{mg} / \mathrm{ml})$ or PBS solution was infused at $0.2 \mu \mathrm{l} / \mathrm{min}$ via a glass micropipette. We selected this region of the brain because npTau has a deleterious effect on newborn neurons of the hippocampus (Bolos et al., 2017b). After 2 weeks, mice were fully anesthetized by an intraperitoneal injection of $50 \mu \mathrm{l}$ of pentobarbital and transcardially perfused with $0.9 \%$ saline. Brains were removed and hippocampi were homogenized in RIPA buffer ( $50 \mathrm{mM}$ Tris- $\mathrm{HCl}$ buffer $\mathrm{pH}=7.4 ; 150 \mathrm{mM} \mathrm{NaCl}$; $1 \%$ Triton; $0.5 \%$ deoxycholate, $0.1 \%$ SDS) containing a mixture of protease inhibitors (Roche, Switzerland). Homogenates were centrifuged, and the proteins present in the supernatants were analyzed by immunoblotting.

\subsection{Cytokine protein array}

Microglia culture media were collected after $2 \mathrm{~h}$ of npTau or PBS treatment. For each group, media, derived from four independent experiments, were pooled in a single tube following the manufacturer's recommendations. The mouse cytokine array (RayBio C-series Mouse Cytokine Antibody Array C1000 Ray Biotech Inc., USA) consisted of cytokine antibodies spotted onto a membrane. Incubations were performed following the manufacturer's instructions and as previously described (Bolos et al., 2018). For each spot, the net density of the gray level was normalized by subtracting the background from the total raw density using the ImageQuant ${ }^{\mathrm{TM}}$ LAS 4000 mini (GE Healthcare, USA) and Image J analysis software Version 1.50i (National Institutes of Health, USA). For each treatment group, the relative level of each cytokine was calculated. Only those cytokines whose levels showed a variation greater than a $25 \%$ as compared to those of the control (PBS, dotted line) are shown in Fig. 4. The levels of the remaining cytokines are provided in Supporting Fig. S1.

\subsection{Quantitative polymerase chain reaction ( $q P C R$ )}

Between 80,000 and 100,000 cells were treated with npTau or PBS for $2 \mathrm{~h}$ in the presence or absence of SB and then quickly rinsed with PBS. Total RNA from culture plates was isolated with the QIAzol Lysis Reagent (Qiagen, Germany) and RNeasy Mini kit (Qiagen, Germany) and treated with DNase I to remove interfering genomic DNA. All samples showed a RNA integrity number (RIN) between 7.9 and 10 . First-strand cDNA was synthesized from $200 \mathrm{ng}$ of total RNA using the High Capacity cDNA Archive Kit (Applied Biosystems, Thermo Fischer, USA). Real-time PCR of selected genes was performed by using TaqMan-probe assays in an $\mathrm{AB}$ fast-7900HT System (Applied Biosystems, Thermo Fischer, USA) under standard operating conditions. The following commercial Taqman probes (Applied Biosystems, Thermo Fisher, USA) were used:

\begin{tabular}{ll}
\hline Il6 & Mm00446190_m1 \\
Mip1 $\alpha$ & Mm00441259_g1 \\
Tnf & Mm00443258_m1 \\
Il1b & Mm00434228_m1 \\
Gapdh & Mm99999915_g1 \\
\hline
\end{tabular}

Three replicates of cDNA were run for each combination of genes and samples. Ct assignment was performed using the Sequence Detection System 2.4 software (Applied Biosystems, Thermo Fisher, USA) to set baseline and threshold parameters. Assay efficiency was assumed to be $100 \%$. Expression levels of target genes in treated cells were normalized to the expression of GAPDH, and results were referred to control samples. Relative changes and statistical values of mRNA expression levels were calculated by the ddCt method, using StatMiner software (Integromics, Perkin Elmer, USA).

\subsection{Immunoblotting}

The levels of p38 MAPK and phospho-p38 MAPK in primary microglia cultures and tissue and; MK2, phospho-MK2, and GAPDH in primary microglia cultures were determined by western blotting. Cells were washed with PBS and then lysed in RIPA buffer and protease inhibitor mixture (Roche, Switzerland). Proteins were then separated on $10 \%$ sodium dodecyl sulfate-polyacrylamide gels before being transferred electrophoretically onto polyvinylidene difluoride membranes (Millipore, Germany). The membranes were blocked for $2 \mathrm{~h}$ with $5 \%$ $(w / v)$ bovine serum albumin in $50 \mathrm{mM}$ of Tris-buffered saline $\mathrm{pH}=8$, containing $0.05 \%(v / v)$ of Tween 20 (TBS-Tween). They were then incubated overnight at $4{ }^{\circ} \mathrm{C}$ with the following: p38 MAPK antibody (\#9212 Cell Signaling 1:1000 dilution); phospho-p38 MAPK (Thr180/ Tyr182) antibody (\#9211 Cell Signaling 1:1000 dilution); MK2 antibody (sc-393,609 SCBT 1:100 dilution); phospho-MK2 (Thr334) antibody (sc-293,140 SCBT 1:1000 dilution); or GAPDH antibody (ab8245 Abcam 1:1000 dilution). Protein expression was detected using HRPconjugated secondary antibodies (1:2000 dilution). For quantification of immunoreactivity, images of blots were analyzed using Image $J$ Version 1.50i (National Institutes of Health, USA). All results were obtained in at least three independent experiments.

In the experiments of Tau dephosphorylation, Tau12 (MAB2241 Millipore 1:300 dilution), PHF-1 (provided by Prof. Davies (Albert Einstein College of Medicine, New York, NY) 1:100 dilution), AT8 (MN1020 Thermo Fisher 1:100 dilution), AT100 (MN1060 Thermo Fisher 1:100 dilution), and AT180 (MN1040 Thermo Fisher 1:100 dilution) were used as primary antibodies. Secondary antibodies were used at 1:2000 dilution. 


\subsection{Statistical analysis}

Statistical analysis was performed with GraphPad Prism software Version 7.0a (USA). We used the term $\mathrm{N}$ in reference to the number of independent experiments that we performed. Each independent experiment encompassed a new cell culture and new treatment per condition described. We performed three replicates per condition. Data were tested by Student's $t$-test or two-way analysis of variance. Post-hoc comparisons were analyzed using Tukey's test. Differences were considered statistically significant when the probability, $p$, of the null hypothesis was $\leq 0.05$. Data are presented as the means \pm S.E.

Supplementary data to this article can be found online at https:// doi.org/10.1016/j.expneurol.2018.08.007.

\section{Acknowledgments}

The authors would like to thank Raquel Cuadros and Esther García for technical assistance.

\section{Funding}

This study was supported by the Spanish Ministry of Economy and Competitiveness (SAF-2014-53040-P (Jesús Ávila)) and the Centro de Investigación Biomédica en Red sobre Enfermedades Neurodegenerativas (CIBERNED, Spain) (Jesús Ávila).

\section{Conflict of interest disclosure}

The authors declare no conflict of interest.

\section{Author contributions}

MB and JA conceived and designed the study; MB and JRP collected the data; MB, JRP and JA analyzed the data; and MB, JRP and JA drafted the manuscript. All authors read and approved the final manuscript.

\section{References}

Adolfsson, O., Pihlgren, M., Toni, N., Varisco, Y., Buccarello, A.L., Antoniello, K., Lohmann, S., Piorkowska, K., Gafner, V., Atwal, J.K., Maloney, J., Chen, M., Gogineni, A., Weimer, R.M., Mortensen, D.L., Friesenhahn, M., Ho, C., Paul, R. Pfeifer, A., Muhs, A., Watts, R.J., 2012. An effector-reduced anti-beta-amyloid (Abeta) antibody with unique abeta binding properties promotes neuroprotection and glial engulfment of Abeta. J Neurosci 32, 9677-9689.

Aloisi, F., 2001. Immune function of microglia. Glia 36, 165-179.

Alonso, A.D., Beharry, C., Corbo, C.P., Cohen, L.S., 2016. Molecular mechanism of prionlike tau-induced neurodegeneration. Alzheimers Dement. 12, 1090-1097.

Andorfer, C., Acker, C.M., Kress, Y., Hof, P.R., Duff, K., Davies, P., 2005. Cell-cycle reentry and cell death in transgenic mice expressing nonmutant human tau isoforms. J. Neurosci. 25, 5446-5454.

Arthur, J.S., Ley, S.C., 2013. Mitogen-activated protein kinases in innate immunity. Nat. Rev. Immunol. 13, 679-692.

Avila, J., Jimenez, J.S., Sayas, C.L., Bolos, M., Zabala, J.C., Rivas, G., Hernandez, F., 2016. Tau structures. Front. Aging Neurosci. 8, 262.

Bachstetter, A.D., Xing, B., de Almeida, L., Dimayuga, E.R., Watterson, D.M., Van Eldik, L.J., 2011. Microglial p38alpha MAPK is a key regulator of proinflammatory cytokine up-regulation induced by toll-like receptor (TLR) ligands or beta-amyloid (Abeta). J. Neuroinflammation 8, 79.

Bachstetter, A.D., Watterson, D.M., Van Eldik, L.J., 2014. Target engagement analysis and link to pharmacodynamic endpoint for a novel class of CNS-penetrant and efficacious p38alpha MAPK inhibitors. J. NeuroImmune Pharmacol. 9, 454-460.

Barone, F.C., Irving, E.A., Ray, A.M., Lee, J.C., Kassis, S., Kumar, S., Badger, A.M., Legos, J.J., Erhardt, J.A., Ohlstein, E.H., Hunter, A.J., Harrison, D.C., Philpott, K., Smith, B.R., Adams, J.L., Parsons, A.A., 2001a. Inhibition of p38 mitogen-activated protein kinase provides neuroprotection in cerebral focal ischemia. Med. Res. Rev. 21, $129-145$.

Barone, F.C., Irving, E.A., Ray, A.M., Lee, J.C., Kassis, S., Kumar, S., Badger, A.M., White, R.F., McVey, M.J., Legos, J.J., Erhardt, J.A., Nelson, A.H., Ohlstein, E.H., Hunter, A.J., Ward, K., Smith, B.R., Adams, J.L., Parsons, A.A., 2001b. SB 239063, a secondgeneration p38 mitogen-activated protein kinase inhibitor, reduces brain injury and neurological deficits in cerebral focal ischemia. J. Pharmacol. Exp. Ther. 296, 312-321.

Bolos, M., Llorens-Martin, M., Jurado-Arjona, J., Hernandez, F., Rabano, A., Avila, J.,
2015. Direct evidence of internalization of tau by microglia in vitro and in vivo. J. Alzheimers Dis. 50, 77-87.

Bolos, M., Llorens-Martin, M., Perea, J.R., Jurado-Arjona, J., Rabano, A., Hernandez, F., Avila, J., 2017a. Absence of CX3CR1 impairs the internalization of Tau by microglia. Mol. Neurodegener. 12, 59.

Bolos, M., Pallas-Bazarra, N., Terreros-Roncal, J., Perea, J.R., Jurado-Arjona, J., Avila, J., Llorens-Martin, M., 2017b. Soluble Tau has devastating effects on the structural plasticity of hippocampal granule neurons. Transl. Psychiatry 7, 1267.

Bolos, M., Perea, J.R., Avila, J., 2017c. Alzheimer's disease as an inflammatory disease. Biomol. Concepts 8, 37-43.

Bolos, M., Perea, J.R., Terreros-Roncal, J., Pallas-Bazarra, N., Jurado-Arjona, J., Avila, J., Llorens-Martin, M., 2018. Absence of microglial CX3CR1 impairs the synaptic integration of adult-born hippocampal granule neurons. Brain Behav. Immun. 68, 76-89.

Bradham, C., McClay, D.R., 2006. p38 MAPK in development and cancer. Cell Cycle 5, 824-828.

Bussiere, T., Hof, P.R., Mailliot, C., Brown, C.D., Caillet-Boudin, M.L., Perl, D.P., Buee, L., Delacourte, A., 1999. Phosphorylated serine422 on tau proteins is a pathological epitope found in several diseases with neurofibrillary degeneration. Acta Neuropathol. 97, 221-230.

Caceres, A., Kosik, K.S., 1990. Inhibition of neurite polarity by tau antisense oligonucleotides in primary cerebellar neurons. Nature 343, 461-463.

Correa, S.A., Eales, K.L., 2012. The role of p38 MAPK and its substrates in neuronal plasticity and neurodegenerative disease. J. Signal. Transduct. 2012, 649079.

Cowan, C.M., Mudher, A., 2013. Are tau aggregates toxic or protective in tauopathies? Front. Neurol. 4, 114.

Culbert, A.A., Skaper, S.D., Howlett, D.R., Evans, N.A., Facci, L., Soden, P.E., Seymour, Z.M., Guillot, F., Gaestel, M., Richardson, J.C., 2006. MAPK-activated protein kinase 2 deficiency in microglia inhibits pro-inflammatory mediator release and resultant neurotoxicity. Relevance to neuroinflammation in a transgenic mouse model of Alzheimer disease. J. Biol. Chem. 281, 23658-23667.

Dewil, M., dela Cruz, V.F., Van Den Bosch, L., Robberecht, W., 2007. Inhibition of p38 mitogen activated protein kinase activation and mutant SOD1(G93A)-induced motor neuron death. Neurobiol. Dis. 26, 332-341.

Diaz-Aparicio, I., Beccari, S., Abiega, O., Sierra, A., 2016. Clearing the corpses: regulatory mechanisms, novel tools, and therapeutic potential of harnessing microglial phagocytosis in the diseased brain. Neural Regen. Res. 11, 1533-1539.

Diaz-Hernandez, M., Gomez-Ramos, A., Rubio, A., Gomez-Villafuertes, R., Naranjo, J.R., Miras-Portugal, M.T., Avila, J., 2010. Tissue-nonspecific alkaline phosphatase promotes the neurotoxicity effect of extracellular tau. J. Biol. Chem. 285, 32539-32548.

Diaz-Hernandez, M., Hernandez, F., Miras-Portugal, M.T., Avila, J., 2015. TNAP plays a key role in neural differentiation as well as in neurodegenerative disorders. Subcell. Biochem. 76, 375-385.

Drubin, D.G., Kirschner, M.W., 1986. Tau protein function in living cells. J. Cell Biol. 103, 2739-2746.

Fa, M., Puzzo, D., Piacentini, R., Staniszewski, A., Zhang, H., Baltrons, M.A., Li Puma, D.D., Chatterjee, I., Li, J., Saeed, F., Berman, H.L., Ripoli, C., Gulisano, W., Gonzalez, J., Tian, H., Costa, J.A., Lopez, P., Davidowitz, E., Yu, W.H., Haroutunian, V., Brown, L.M., Palmeri, A., Sigurdsson, E.M., Duff, K.E., Teich, A.F., Honig, L.S., Sierks, M., Moe, J.G., D'Adamio, L., Grassi, C., Kanaan, N.M., Fraser, P.E., Arancio, O., 2016. Extracellular tau oligomers produce an immediate impairment of LTP and memory. Sci. Rep. 6, 19393.

Fox, L.M., William, C.M., Adamowicz, D.H., Pitstick, R., Carlson, G.A., Spires-Jones, T.L., Hyman, B.T., 2011. Soluble tau species, not neurofibrillary aggregates, disrupt neural system integration in a tau transgenic model. J. Neuropathol. Exp. Neurol. 70, 588-595.

Gerson, J.E., Castillo-Carranza, D.L., Kayed, R., 2014. Advances in therapeutics for neurodegenerative tauopathies: moving toward the specific targeting of the most toxic tau species. ACS Chem. Neurosci. 5, 752-769.

Ghasemlou, N., Lopez-Vales, R., Lachance, C., Thuraisingam, T., Gaestel, M., Radzioch, D., David, S., 2010. Mitogen-activated protein kinase-activated protein kinase 2 (MK2) contributes to secondary damage after spinal cord injury. J. Neurosci. 30, 13750-13759.

Gomez-Ramos, A., Abad, X., Lopez Fanarraga, M., Bhat, R., Zabala, J.C., Avila, J., 2004 Expression of an altered form of tau in Sf9 insect cells results in the assembly of polymers resembling Alzheimer's paired helical filaments. Brain Res. 1007, 57-64.

Gomez-Ramos, A., Diaz-Hernandez, M., Cuadros, R., Hernandez, F., Avila, J., 2006. Extracellular tau is toxic to neuronal cells. FEBS Lett. 580, 4842-4850.

Gonzalez-Scarano, F., Baltuch, G., 1999. Microglia as mediators of inflammatory and degenerative diseases. Annu. Rev. Neurosci. 22, 219-240.

Grundke-Iqbal, I., Iqbal, K., Tung, Y.C., Quinlan, M., Wisniewski, H.M., Binder, L.I., 1986. Abnormal phosphorylation of the microtubule-associated protein tau (tau) in Alzheimer cytoskeletal pathology. Proc. Natl. Acad. Sci. U. S. A. 83, 4913-4917.

Haass, C., Selkoe, D.J., 2007. Soluble protein oligomers in neurodegeneration: lessons from the Alzheimer's amyloid beta-peptide. Nat. Rev. Mol. Cell Biol. 8, 101-112.

Hanisch, U.K., 2002. Microglia as a source and target of cytokines. Glia 40, 140-155.

Hasegawa, M., Jakes, R., Crowther, R.A., Lee, V.M., Ihara, Y., Goedert, M., 1996. Characterization of mAb AP422, a novel phosphorylation-dependent monoclonal antibody against tau protein. FEBS Lett. 384, 25-30.

Hoffmann, R., Lee, V.M., Leight, S., Varga, I., Otvos Jr., L., 1997. Unique Alzheimer's disease paired helical filament specific epitopes involve double phosphorylation at specific sites. Biochemistry 36, 8114-8124.

Holmes, B.B., Diamond, M.I., 2014. Prion-like properties of Tau protein: the importance of extracellular Tau as a therapeutic target. J. Biol. Chem. 289, 19855-19861.

Hyman, B.T., 2014. Tau propagation, different tau phenotypes, and prion-like properties of tau. Neuron 82, 1189-1190. 
Ishihara, T., Hong, M., Zhang, B., Nakagawa, Y., Lee, M.K., Trojanowski, J.Q., Lee, V.M., 1999. Age-dependent emergence and progression of a tauopathy in transgenic mice overexpressing the shortest human tau isoform. Neuron 24, 751-762.

Ishihara, T., Zhang, B., Higuchi, M., Yoshiyama, Y., Trojanowski, J.Q., Lee, V.M., 2001 Age-dependent induction of congophilic neurofibrillary tau inclusions in tau transgenic mice. Am. J. Pathol. 158, 555-562.

Jicha, G.A., Lane, E., Vincent, I., Otvos Jr., L., Hoffmann, R., Davies, P., 1997. A conformation- and phosphorylation-dependent antibody recognizing the paired helical filaments of Alzheimer's disease. J. Neurochem. 69, 2087-2095.

Kreutzberg, G.W., 1996. Microglia: a sensor for pathological events in the CNS. Trends Neurosci. 19, 312-318.

Le Corre, S., Klafki, H.W., Plesnila, N., Hubinger, G., Obermeier, A., Sahagun, H., Monse, B., Seneci, P., Lewis, J., Eriksen, J., Zehr, C., Yue, M., McGowan, E., Dickson, D.W., Hutton, M., Roder, H.M., 2006. An inhibitor of tau hyperphosphorylation prevents severe motor impairments in tau transgenic mice. Proc. Natl. Acad. Sci. U. S. A. 103, 9673-9678.

Lee, J.K., Kim, N.J., 2017. Recent advances in the inhibition of p38 MAPK as a potential strategy for the treatment of Alzheimer's disease. Molecules 22.

Leyns, C.E.G., Holtzman, D.M., 2017. Glial contributions to neurodegeneration in tauopathies. Mol. Neurodegener. 12, 50.

Maphis, N., Xu, G., Kokiko-Cochran, O.N., Jiang, S., Cardona, A., Ransohoff, R.M., Lamb, B.T., Bhaskar, K., 2015. Reactive microglia drive tau pathology and contribute to the spreading of pathological tau in the brain. Brain 138, 1738-1755.

McDonald, D.R., Bamberger, M.E., Combs, C.K., Landreth, G.E., 1998. Beta-Amyloid fibrils activate parallel mitogen-activated protein kinase pathways in microglia and THP1 monocytes. J. Neurosci. 18, 4451-4460.

McGeer, P.L., Schulzer, M., McGeer, E.G., 1996. Arthritis and anti-inflammatory agents as possible protective factors for Alzheimer's disease: a review of 17 epidemiologic studies. Neurology 47, 425-432.

Medina, M., Avila, J., 2014a. Is tau a prion-like protein? J. Alzheimers Dis. 40, S1-S3 Suppl 1.

Medina, M., Avila, J., 2014b. The role of extracellular Tau in the spreading of neurofibrillary pathology. Front. Cell. Neurosci. 8, 113.

Medina, M., Montejo De Garcini, E., Avila, J., 1995. The role of tau phosphorylation in transfected COS-1 cells. Mol. Cell. Biochem. 148, 79-88.

Medina, M., Hernandez, F., Avila, J., 2016. New features about tau function and dysfunction. Biomol. Ther. 6 .

Mirbaha, H., Holmes, B.B., Sanders, D.W., Bieschke, J., Diamond, M.I., 2015. Tau trimers are the minimal propagation unit spontaneously internalized to seed intracellular aggregation. J. Biol. Chem. 290, 14893-14903.

Munoz, L., Ralay Ranaivo, H., Roy, S.M., Hu, W., Craft, J.M., McNamara, L.K., Chico, L.W., Van Eldik, L.J., Watterson, D.M., 2007. A novel p38 alpha MAPK inhibitor suppresses brain proinflammatory cytokine up-regulation and attenuates synaptic dysfunction and behavioral deficits in an Alzheimer's disease mouse model. J. Neuroinflammation 4, 21 .

Odawara, T., Iseki, E., Kosaka, K., Akiyama, H., Ikeda, K., Yamamoto, T., 1995. Investigation of tau-2 positive microglia-like cells in the subcortical nuclei of human neurodegenerative disorders. Neurosci. Lett. 192, 145-148.

Perez, M., Cuadros, R., Hernandez, F., Avila, J., 2016. Secretion of full-length tau or tau fragments in a cell culture model. Neurosci. Lett. 634, 63-69.

Piao, C.S., Kim, J.B., Han, P.L., Lee, J.K., 2003. Administration of the p38 MAPK inhibitor SB203580 affords brain protection with a wide therapeutic window against focal ischemic insult. J. Neurosci. Res. 73, 537-544.

Ransohoff, R.M., 2016. How neuroinflammation contributes to neurodegeneration. Science 353, 777-783.
Ransohoff, R.M., El Khoury, J., 2016. Microglia in health and disease. Cold Spring Harb. Perspect. Biol. 8, a020560.

Roy, S.M., Grum-Tokars, V.L., Schavocky, J.P., Saeed, F., Staniszewski, A., Teich, A.F. Arancio, O., Bachstetter, A.D., Webster, S.J., Van Eldik, L.J., Minasov, G., Anderson, W.F., Pelletier, J.C., Watterson, D.M., 2015. Targeting human central nervous system protein kinases: an isoform selective p38alphaMAPK inhibitor that attenuates disease progression in Alzheimer's disease mouse models. ACS Chem. Neurosci. 6, 666-680.

Scheltens, P., Prins, N., Lammertsma, A., Yaqub, M., Gouw, A., Wink, A.M., Chu, H.M., van Berckel, B.N.M., Alam, J., 2018. An exploratory clinical study of p38alpha kinase inhibition in Alzheimer's disease. Ann. Clin. Transl. Neurol. 5, 464-473.

Selkoe, D.J., Ihara, Y., Salazar, F.J., 1982. Alzheimer's disease: insolubility of partially purified paired helical filaments in sodium dodecyl sulfate and urea. Science 215, 1243-1245.

Sierra, A., Tremblay, M.E., Wake, H., 2014. Never-resting microglia: physiological roles in the healthy brain and pathological implications. Front. Cell. Neurosci. 8, 240.

Spires-Jones, T.L., Stoothoff, W.H., de Calignon, A., Jones, P.B., Hyman, B.T., 2009. Tau pathophysiology in neurodegeneration: a tangled issue. Trends Neurosci. 32, 150-159.

Sun, A., Liu, M., Nguyen, X.V., Bing, G., 2003. P38 MAP kinase is activated at early stages in Alzheimer's disease brain. Exp. Neurol. 183, 394-405.

Tomlinson, B.E., Blessed, G., Roth, M., 1970. Observations on the brains of demented old people. J. Neurol. Sci. 11, 205-242.

Tseng, J.H., Xie, L., Song, S., Xie, Y., Allen, L., Ajit, D., Hong, J.S., Chen, X., Meeker, R.B., Cohen, T.J., 2017. The deacetylase HDAC6 mediates endogenous neuritic tau pathology. Cell Rep. 20, 2169-2183.

Underwood, D.C., Osborn, R.R., Bochnowicz, S., Webb, E.F., Rieman, D.J., Lee, J.C., Romanic, A.M., Adams, J.L., Hay, D.W., Griswold, D.E., 2000. SB 239063, a p38 MAPK inhibitor, reduces neutrophilia, inflammatory cytokines, MMP-9, and fibrosis in lung. Am. J. Phys. Lung Cell. Mol. Phys. 279, L895-L902.

Wang, S., Zhang, C., Sheng, X., Zhang, X., Wang, B., Zhang, G., 2014. Peripheral expression of MAPK pathways in Alzheimer's and Parkinson's diseases. J. Clin. Neurosci. $21,810-814$

Watterson, D.M., Grum-Tokars, V.L., Roy, S.M., Schavocky, J.P., Bradaric, B.D., Bachstetter, A.D., Xing, B., Dimayuga, E., Saeed, F., Zhang, H., Staniszewski, A. Pelletier, J.C., Minasov, G., Anderson, W.F., Arancio, O., Van Eldik, L.J., 2013. Development of novel in vivo chemical probes to address CNS protein kinase involvement in synaptic dysfunction. PLoS One 8, e66226.

Whitehouse, P.J., Price, D.L., Struble, R.G., Clark, A.W., Coyle, J.T., Delon, M.R., 1982. Alzheimer's disease and senile dementia: loss of neurons in the basal forebrain. Science 215, 1237-1239.

Wittmann, C.W., Wszolek, M.F., Shulman, J.M., Salvaterra, P.M., Lewis, J., Hutton, M., Feany, M.B., 2001. Tauopathy in Drosophila: neurodegeneration without neurofibrillary tangles. Science 293, 711-714.

Xing, B., Bachstetter, A.D., Van Eldik, L.J., 2011. Microglial p38alpha MAPK is critical for LPS-induced neuron degeneration, through a mechanism involving TNFalpha. Mol. Neurodegener. 6, 84.

Yamada, K., Holth, J.K., Liao, F., Stewart, F.R., Mahan, T.E., Jiang, H., Cirrito, J.R., Patel, T.K., Hochgrafe, K., Mandelkow, E.M., Holtzman, D.M., 2014. Neuronal activity regulates extracellular Tau in vivo. J. Exp. Med. 211, 387-393.

Young, P.R., McLaughlin, M.M., Kumar, S., Kassis, S., Doyle, M.L., McNulty, D., Gallagher, T.F., Fisher, S., McDonnell, P.C., Carr, S.A., Huddleston, M.J., Seibel, G., Porter, T.G., Livi, G.P., Adams, J.L., Lee, J.C., 1997. Pyridinyl imidazole inhibitors of p38 mitogenactivated protein kinase bind in the ATP site. J. Biol. Chem. 272, 12116-12121.

Zhu, X., Lee, H.G., Raina, A.K., Perry, G., Smith, M.A., 2002. The role of mitogen-activated protein kinase pathways in Alzheimer's disease. Neurosignals 11, 270-281. 